\section{Biallelic mutations in the SARS2 gene presenting as congenital sideroblastic anemia}

Mitochondrial disorders (MID) are a clinically heterogeneous group of metabolic disorders characterized by impaired mitochondrial function. They can be caused by mutations in either mitochondrial DNA (mtDNA) or nuclear DNA encoding mitochondrial proteins. In addition to energy deficiency, consequences of mitochondrial dysfunction can include excessive reactive oxygen species (ROS) production, defect in heme synthesis, aberrant calcium handling, and apoptosis dysregulation, all of which contribute to the pathogenesis of MID. ${ }^{1}$ MID are usually multisystemic disorders, affecting various organs but only a few of them such as congenital sideroblastic anemias (CSA) display major erythroid abnormalities. CSA is a series of rare, heterogeneous disorders characterized by pathological iron accumulation in the mitochondria of erythroblasts and presence of ring sideroblasts in the bone marrow at varying degrees. ${ }^{2,3}$ So far, nearly two-thirds of CSA cases have been attributed to a mutation in a specific gene or genes. ${ }^{4}$

Here we report the original case of a patient presenting with CSA, in which we identified biallelic mutations of the seryl-tRNA synthetase 2 (SARS2) gene.

The affected child was a first-born son to a non-consanguineous healthy couple with no neonatal antecedents and no familial medical history. His younger sister is healthy (Figure 1A). He presented at the age of 3 years with chronic renal failure associated with cerebellar atrophy, hypertension, failure to thrive, polyuria and polydipsia. Kidney biopsy revealed a tubulointerstitial nephritis. Metabolic workup showed hyperlactatemia and abnormal acylcarnitine profile, findings suggesting of a MID. Blood tests concomitantly revealed a non-regenerative anemia associated with mild hemolysis, which was refractory to erythropoietin (EPO) treatment and required monthly blood transfusions. Negative direct antiglobulin test ruled out the presence of an immune disorder. No abnormal hemoglobin $(\mathrm{Hb})$ was detected on electrophoresis profile and the levels of pyruvate kinase and G6PD enzymes were in normal values. Peripheral blood smear showed the presence of about $20 \%$ spherocytes (Online Supplementary Figure S1A). Accordingly, osmotic gradient ektacytometry revealed reduced erythrocyte deformability, supporting the diagnosis of hereditary spherocytosis (Online Supplementary Figure S1B). Bone marrow aspiration showed no signs of dyserythropoiesis but a small contingent of ring sideroblasts $(5 \%)$ was observed (Figure 1B). Iron and transferrin serum levels were in normal ranges but ferritin level and transferrin saturation were elevated. Patient's laboratory data are detailed in the Online Supplementary Table S1.

Patient's condition progressively deteriorated leading to terminal end-stage renal disease and very severe anemia requiring weekly blood transfusions. He ultimately died of pulmonary hypertension at the age of 5 years.

Whole exome sequencing performed on the proband and

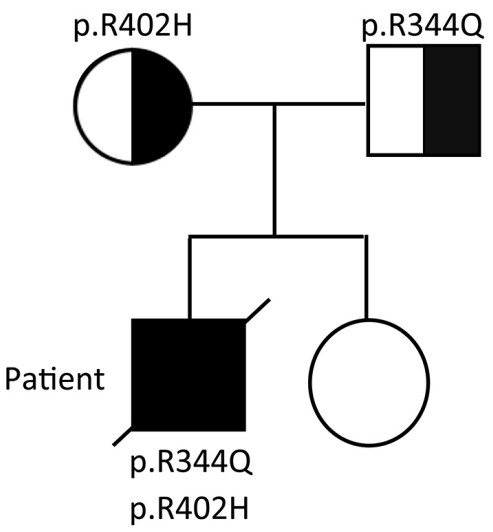

B

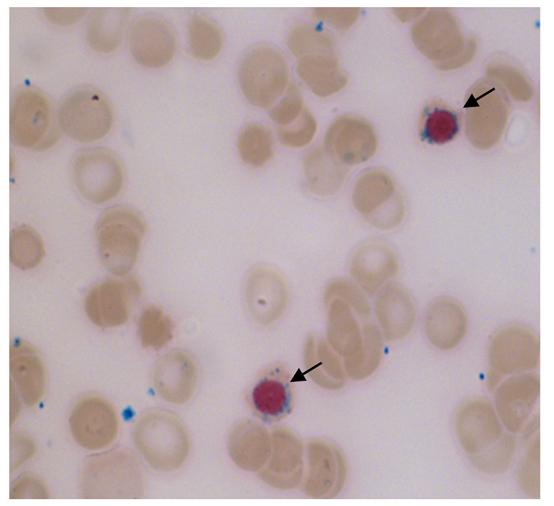

C

SARS2

R344

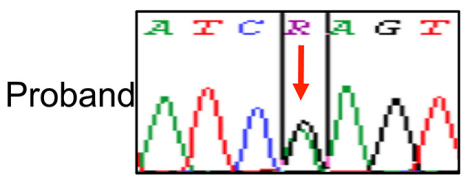

Mother

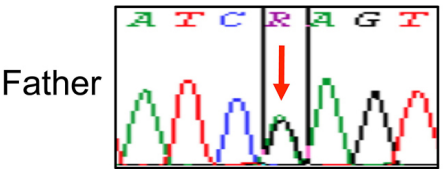

$\mathrm{R} 402$
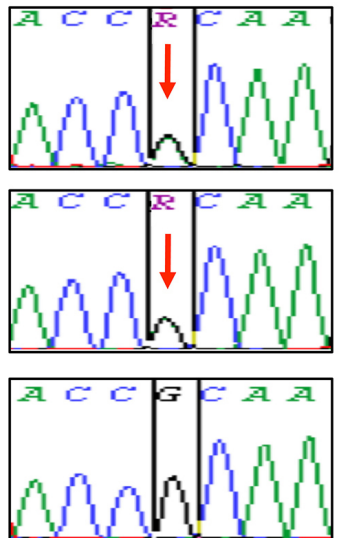

Figure 1. Clinical and molecular findings of the proband. (A) Pedigree and segregation of the pathogenic SARS2 variants in the reported family. (B) Bone marrow aspiration from the proband showing the presence of ring sideroblasts on iron stain. (C) Sanger sequencing validation of SARS2 c.1031 G>A ( $p . R 344 Q$ ) and c.1205 G>A (p.R402H) mutations detected by next generation sequencing. Red arrows indicate the position of the nucleotide's substitution. 
his parents identified a de novo heterozygous nonsense mutation (c. 6037C > T p.Q2013X) in the SPTB gene. This gene encodes a member of the spectrin family, involved in the stability of erythrocytes membranes. Mutations in this gene have been associated with hereditary spherocytosis and hemolytic anemia. ${ }^{5}$ While most symptoms of the proband were consistent with hereditary spherocytosis, the non-regenerative anemia, together with the presence of ring sideroblasts and extra-hematological signs remained unexplained, suggesting that additional mutations were involved in his phenotype. According to this hypothesis, we identified in the proband two compound heterozygous missense variants (c.1031 G>A p.R344Q and c.1205 G>A p.R402H) in the SARS2 gene (cDNA accession number: NM_017827.4, transcript ID: ENST00000221431), each inherited from one of two parents. SARS2 is an ubiquitously expressed nuclear gene encoding a mitochondrial aminoacyl-tRNA synthetase (mtaaRS) whose function is to catalyze the specific ligation of serine to two mitochondrial tRNA isoacceptors: tRNA $^{\text {Ser(AGY) }}$ and tRNA ${ }^{\text {Ser(UCN) }{ }^{6}}$ Both $S A R S 2$ variants were reported to be rare in public human genetic variant databases (GnomAD_exomes MAF: $\mathrm{T}=0.000012$ and $\mathrm{T}=0.000008$ respectively). As they affect highly conserved amino acid residues located in the core catalytic domain of the protein and are predicted to be damaging by in silico softwares, biallelic SARS2 mutations identified in the proband were very likely to result in loss-of function.
Sanger validation of the mutations in SPTB and SARS2 are shown in the Online Supplementary Figure S1C and Figure 1C.

Missense mutations in the SARS2 gene (p.V223M, p.D390G and p.R402H) have been reported so far in six individuals from four distinct families who presented a specific MID characterized by hyperuricemia, pulmonary hypertension, renal failure in infancy and alkalosis and designated as HUPRA syndrome. ${ }^{7-9}$ Another SARS2 variant (homozygous splicing mutation in exon 14) was reported in a single case suffering from progressive spastic paresis instead of HUPRA syndrome, ${ }^{10}$ in agreement with the known phenotypic heterogeneity of mutations involving mitochondrial machinery.

Interestingly, a careful review of the published cases of HUPRA syndrome revealed that five of six were anemic with a $\mathrm{Hb}$ level varying from 4.8 to $9 \mathrm{~g} / \mathrm{dL}$ (Online Supplementary Table S2). However, it appears that anemia associated with HUPRA syndrome has never been investigated mechanistically. We therefore hypothesized that SARS2 plays an important role in erythropoiesis. As all SARS2 variants associated with HUPRA syndrome were expected to result in loss-of function, ${ }^{7-9}$ we used an in vitro $S A R S 2$ knockdown model to explore the consequences of $S A R S 2$ inactivation on erythropoiesis.

In a widely used primary cell culture system that recapitulates erythropoiesis, $\mathrm{CD} 34^{+}$were isolated from unrelated cord blood and expanded for 5 days in a medium contain-

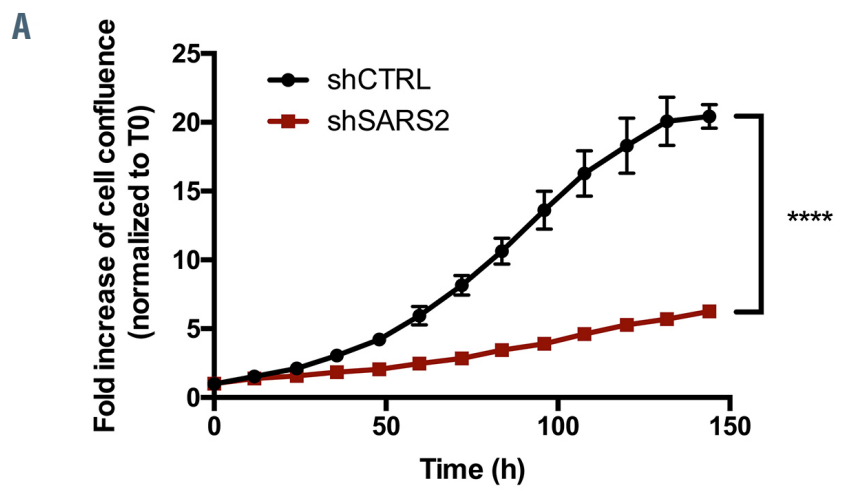

B

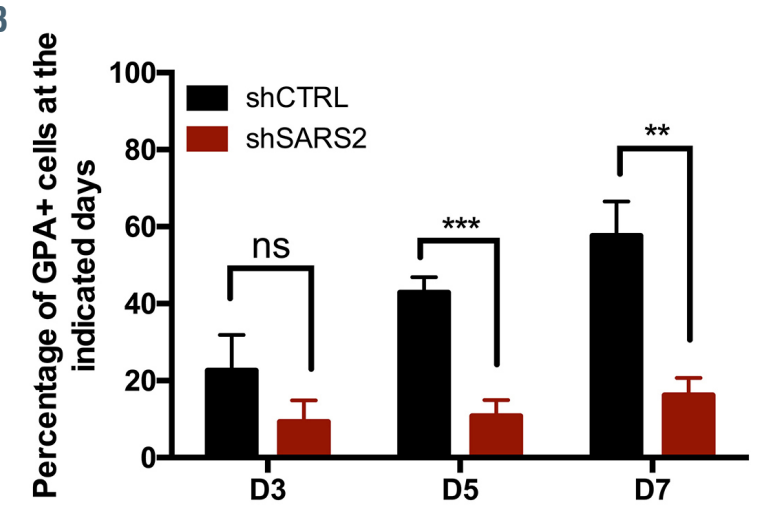

C

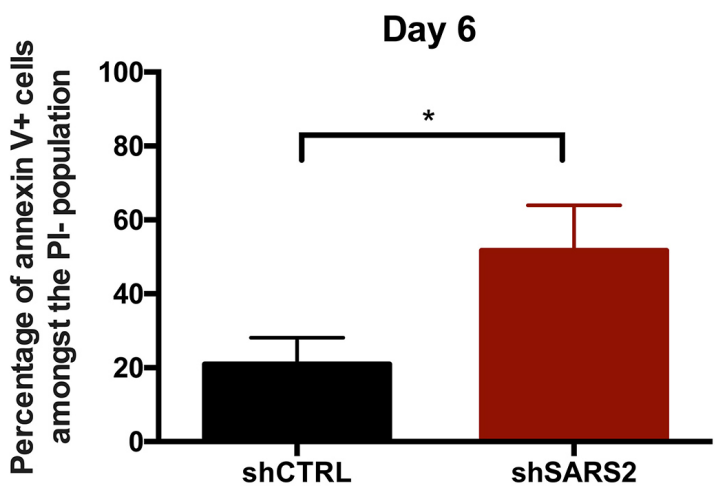

Figure 2. SARS2 depletion in early erythroblasts results in decreased proliferation rate and differentiation arrest due to increased apoptosis. (A) At day 5 (D5) of the first phase of culture, primary erythroid progenitors were transduced with scramble short hairpin RNA (shCTRL) or short hairpin RNA (shRNA) specifically targeting SARS2 (shSARS2). 48 hours later, cell proliferation of transduced cells was monitored for 150 hours by real-time videomicroscopy using the Incucyte ${ }^{8}$ system. Results are represented as the fold increase of cell confluence normalized to TO. Error bars represent standard deviation (SD) from mean of 3 technical replicates. Data are representative of 3 independent experiments. (B) At the indicated days of the second phase of culture, shCTRL-and shSARS2-transduced cells were stained with an antibody directed against the GPA erythroid marker. The percentage of GPA positive cells was then assessed by flow cytometry. Error bars represent SD from mean of 3 independent experiments. (C) At day 6 (D6) of the second phase of culture, shCTRL- and shSARS2-transduced cells were stained with an antibody directed against Annexin $\mathrm{V}$ and with propidium iodide (PI). The percentage of apoptotic cells, defined as the percentage of the Annexin $\checkmark$ positive cells amongst the PI negative population was assessed by flow cytometry. Error bars represent SD from mean of 3 independent experiments. $P$-values are determined by two-tailed $t$-test. ns: not significant; ${ }^{2} P<0.05 ; * * P<0.01 ; * * * P<0.001 ; * * * * P<0.0001$. 
ing $100 \mathrm{ng} / \mathrm{mL}$ interleukin 6 (IL-6), $10 \mathrm{ng} / \mathrm{mL}$ IL-3 and 50 $\mathrm{ng} / \mathrm{mL}$ SCF in Iscove's Modified Dulbecco's medium supplemented with $15 \%$ BIT 9500. Cells were then transduced with green fluorescent protein (GFP) lentiviruses expressing scramble short hairpin RNA (shRNA) or shRNA directed against SARS2 (knockdown efficiency of 70\%). Forty-eight hours later, GFP positive cells were sorted and cultured with the differentiation medium containing $10 \mathrm{ng} / \mathrm{mL}$ IL-3, $50 \mathrm{ng} / \mathrm{mL} \mathrm{SCF}$ and $2 \mathrm{U} / \mathrm{mL}$ of EPO to undergo terminal erythroid differentiation.

$S A R S 2$ depletion in erythroid progenitors resulted in drastic decrease of cell proliferation (Figure 2A) and delayed erythroid differentiation (Figure $2 \mathrm{~B}$ ). In addition, an increased apoptotic rate was observed in SARS2-depleted cells (Figure 2C). As SARS2 is involved in mitochondrial protein synthesis, we wanted to evaluate whether increased apoptosis was related to mitochondrial dysfunction. Accordingly, we measured mitochondrial membrane potential by flow cytometry and assessed the expression levels of activated caspases 3 and 9 by western blot. We observed a profound mitochondrial depolarization in a subset of SARS2-depleted cells (Figure 3A) associated with a cleavage of both caspase 3 and caspase 9 (Figure 3B). Those results indicate that the increased apoptosis reported in SARS2-depleted erythroblasts resulted at least partly, from mitochondrial perturbations. While mutations in mt-aaRS are expected to affect mitochondrial translation system, levels of COXII and ATP8, two mtDNA-encoded subunits of the mitochondrial respiratory chain complex IV and V respectively were found not to be affected in SARS2depleted cells (Figure 3C). In addition, no excessive production of ROS was detected (Online Supplementary Figure

A
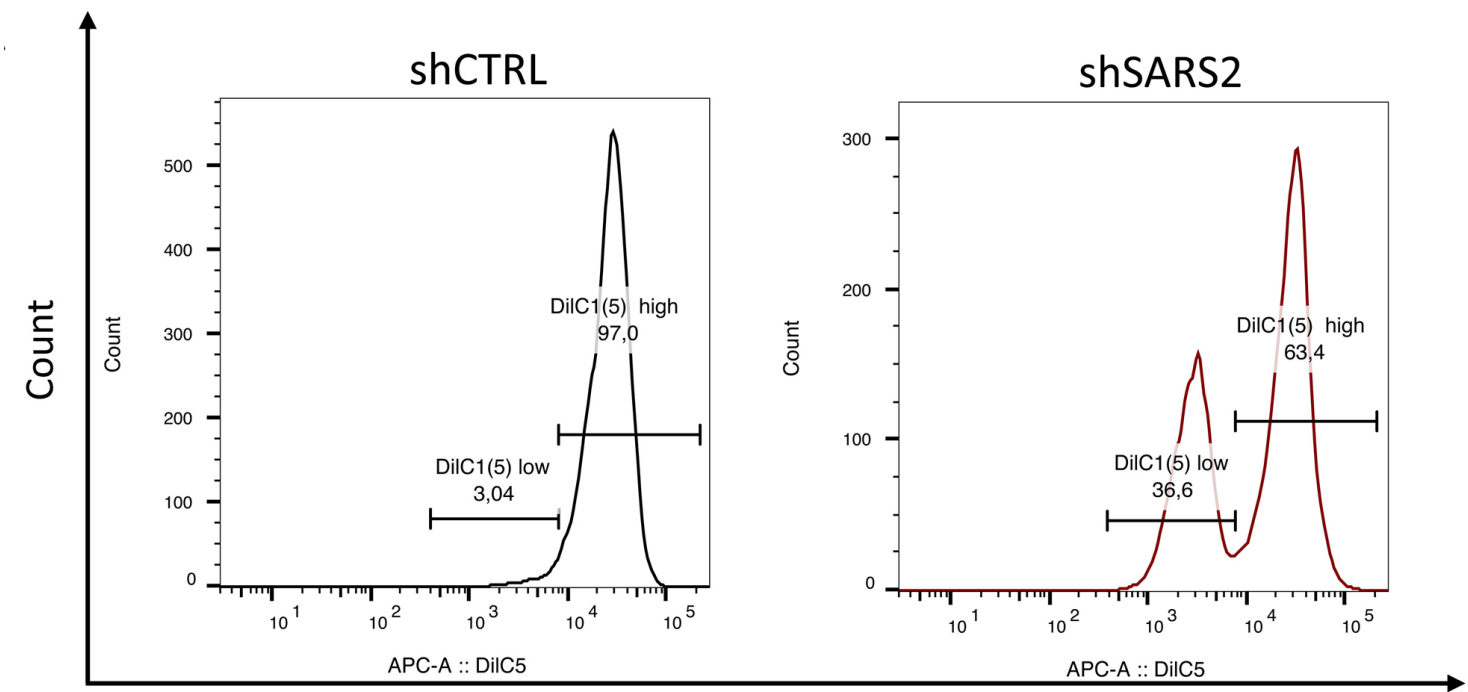

DilC1(5) APC

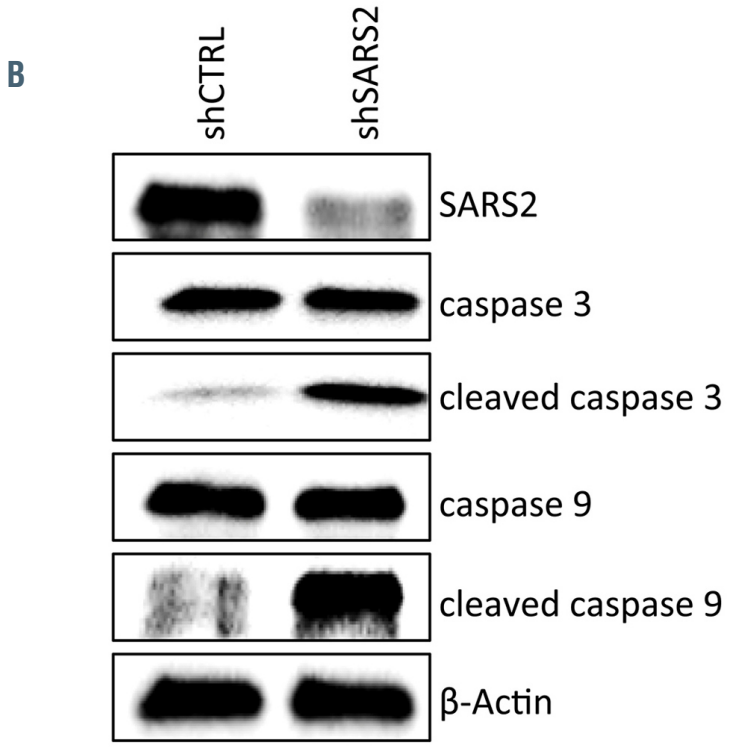

C

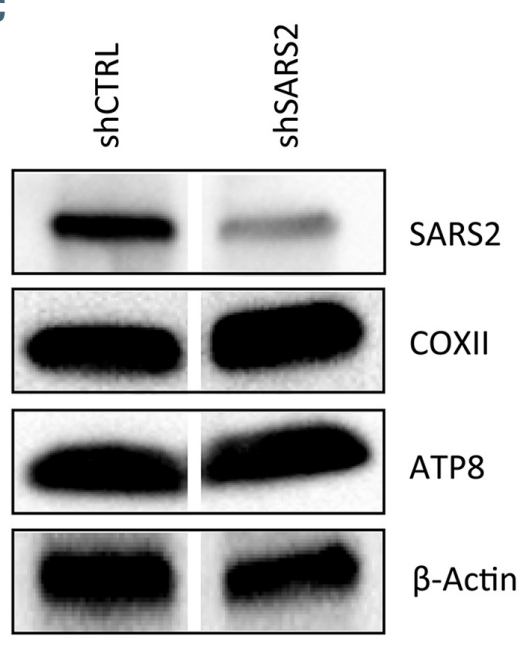

Figure 3. Increased apoptosis in SARS2-depleted cells is mitochondria-mediated. (A) At day 4 of the second phase of culture, mitochondrial membrane potential was assessed by flow cytometry in scramble short hairpin RNA (shCTRL) and shSARS2-transduced cells after staining with the potentiometric DilC1(5) fluorescent dye. The percentage of depolarizing cells, defined as the Dilc1(5) low population was then assessed by flow cytometry. Data are representative of 2 independent experiments. (B) Western blot showing SARS2, cleaved caspase 3 and cleaved caspase 9 expression levels in shCTRL-and shSARS2-transduced cell at day 4 of the second phase of culture. Proteins levels were compared to $\beta$-actin expression. Data are representative of 2 independent experiments. (C) Representative western blot showing SARS2, COXII and ATP8 expression levels in shCTRL-and shSARS2-transduced cells at day 4 of the second phase of culture. Proteins levels were compared to $\beta$-actin expression. 
S1D). Nonetheless, the absence of oxidative phosphorylation or mitochondrial protein synthesis defect observed in the context of SARS2 inactivation is in accordance with the lack of clear biochemical phenotypes previously reported in skin fibroblasts and myoblasts from most of mutant mtaaRS patients. ${ }^{6}$

Despite their common biochemical role in mitochondrial protein synthesis, mutations of the mt-aaRS family have been correlated to a wide range of clinical manifestations. To date, three mt-aaRS defects affecting the tyrosyl-tRNA synthetase 2 (YARS2), ${ }^{11}$ the leucyl-tRNA synthetase 2 $(L A R S 2)^{12}$ and the isoleucyl-tRNA synthetase $2(\text { IARS2 })^{3}$ genes have been associated with erythroid disorders corresponding to CSA.

It is now well established that mitochondria are involved in erythroid cells homeostasis through multiple ways, such as heme synthesis, apoptosis, and cell differentiation following transient caspase activation. Alteration of these mitochondrial functions can therefore lead to a variety of erythroid disorders. ${ }^{13}$ For instance, it has been reported that alteration of physiological pathways involving caspases and mitochondria in myelodysplastic syndromes leads to abnormal activation of a mitochondria-mediated apoptotic pathway in erythroid cells and therefore to an ineffective erythropoiesis. ${ }^{14}$ Accordingly, our results suggest that the non-regenerative anemia observed in the SARS2-mutated patients may be related to an exacerbation of the mitochondria-mediated apoptotic pathway. However, the reason why only few defects in mt-aaRS including SARS2 result in perturbed erythropoiesis remains to be elucidated. With regard to tissue specificity of the different mt-aaRS mutations, one can hypothesize that non-translational functions only crucial in some tissues or cells are specific to each $\mathrm{mt}$-aaRS. It is also possible that the remaining residual activity of some mt-aaRS is sufficient to maintain mitochondrial function in most cell types but not in specific tissues.

In summary, our results strongly suggest that $S A R S 2$ is a new gene involved in CSA, although concomitant hereditary spherocytosis, through chronic stress erythropoiesis, has potentially exacerbated the hematological phenotype in the present case. We therefore consider that SARS2 should be added to the list of genes which have to be explored in patients presenting with sideroblastic anemia in early childhood. Our study also emphasizes the relevance of performing next generation sequencing in patients presenting with severe undiagnosed anemia associated with extra hematological signs to identify new key actors of physiological erythropoiesis.

Elia Colin, ${ }^{1}$ Geneviève Courtois, ${ }^{1}$ Chantal Brouzes, ${ }^{2}$ Juliette Pulman, ${ }^{3}$ Marion Rabant, ${ }^{4}$ Agnès Rötig, ${ }^{3}$ Hélène Taffin, ${ }^{5}$ Mathilde Lion-Lambert, 5 Sylvie Fabrega, ${ }^{6}$ Lydie Da Costa, Mariane De Montalembert, ${ }^{8}$ Rémi Salomon, ${ }^{5}$ Olivier Hermine ${ }^{9}$ and Lucile Couronné

${ }^{1}$ Laboratory of Cellular and Molecular Mechanisms of Hematological Disorders and Therapeutic Implications, INSERM U1163, Imagine Institute, University of Paris, Laboratory of Excellence GR-Ex; ${ }^{2}$ Hematology Laboratory, Hôpital Necker-Enfants Malades, Assistance publique-Hôpitaux de Paris (AP-HP); ${ }^{3}$ Laboratory for Genetics of Mitochondrial Disorders, INSERM U1163, Imagine Institute, University of Paris; ${ }^{4}$ Department of Pathology, Hôpital Necker - Enfants Malades, Assistance Publique-Hôpitaux de Paris, University of Paris; ${ }^{5}$ Department of Pediatric Nephrology, MARHEA, Hôpital Necker - Enfants Malades, Assistance Publique-Hôpitaux de Paris (AP-HP); ${ }^{6}$ VVTG platform, SFR Necker; ${ }^{7}$ Hematology Laboratory, Robert Debré Hospital, Assistance Publique-Hôpitaux de Paris (AP-HP), University of Paris, Laboratory of Excellence GR-Ex;
${ }^{8}$ Department of General Pediatrics and Pediatric Infectious Diseases, Reference Center for Sickle Cell Disease, Hôpital Necker - Enfants Malades, Assistance Publique-Hôpitaux de Paris (AP-HP), Université de Paris; ${ }^{9}$ Hematology Department, Hôpital Necker - Enfants Malades, Assistance Publique - Hôpitaux de Paris (APHP), Laboratory of Cellular and Molecular Mechanisms of Hematological Disorders and Therapeutical Implications, INSERM U1163, Imagine Institute, University of Paris, Laboratory of Excellence GR-Ex and ${ }^{10}$ Laboratory of Onco-Hematology, Hôpital Necker - Enfants Malades, Assistance Publique - Hôpitaux de Paris (APHP), Laboratory of Cellular and Molecular Mechanisms of Hematological Disorders and Therapeutical Implications, INSERM U1163, Imagine Institute, University of Paris, Laboratory of Excellence GR-Ex, Paris, France

Correspondence:

ELIACOLIN - elia.colin@hotmail.fr

doi:10.3324/haematol.2021.279138

Received: May 11, 2021.

Accepted: July 26, 2021

Pre-published: August 19, 2021

Disclosures: no conflicts of interest to disclose.

Contributions: EC, OH and LC designed the study; HT, ML-L, $M d M, L D C$ and $R S$ were responsible for patient management and collection of clinical and laboratory data; MR conducted histopathological analyses; EC, GC and JP designed experiments; EC performed experiments; EC and LC analyzed data and co-wrote the manuscript. All the authors reviewed the manuscript and approved the final version.

Funding: this study was supported by grants from Laboratory of Excellence GR-Ex, reference ANR-11-LABX-0051. The labex GR$E x$ is funded by the IdEx program "Investissements d'avenir" of the French National Research Agency, reference ANR-18-IDEX-0001.

\section{References}

1. El-Hattab AW, Scaglia F. Mitochondrial cytopathies. Cell Calcium. 2016; 60(3):199-206

2. Long Z, Li H, Du Y, Han B. Congenital sideroblastic anemia: advances in gene mutations and pathophysiology. Gene. 2018;668:182-189.

3. Barcia G, Pandithan D, Ruzzenente B, et al. Biallelic IARS2 mutations presenting as sideroblastic anemia. Haematologica. 2020;106(4):1220-1225.

4. Ducamp S, Fleming MD. The molecular genetics of sideroblastic anemia. Blood. 2019;133(1):59-69.

5. He B-J, Liao L, Deng Z-F, et al. Molecular genetic mechanisms of hereditary spherocytosis: current perspectives. Acta Haematol. 2018;139(1):6066.

6. Diodato D, Ghezzi D, Tiranti V. The mitochondrial aminoacyl tRNA synthetases: genes and syndromes. Int J Cell Biol. 2014;2014:787956.

7. Belostotsky R, Ben-Shalom E, Rinat C, et al. Mutations in the mitochondrial Seryl-tRNA synthetase cause hyperuricemia, pulmonary hypertension, renal failure in infancy and alkalosis, HUPRA syndrome. Am J Hum Genet. 2011;88(2):193-200

8. Rivera H, Martín-Hernández E, Delmiro A, et al. A new mutation in the gene encoding mitochondrial seryl-tRNA synthetase as a cause of HUPRA syndrome. BMC Nephrol. 2013;14:195.

9. Zhou Y, Zhong C, Yang Q, et al. Novel SARS2 variants identified in a Chinese girl with HUPRA syndrome. Mol Genet Genomic Med. 2021; 9(4):e1650.

10. Linnankivi T, Neupane N, Richter U, Isohanni P, Tynismaa H. Splicing defect in mitochondrial seryl-tRNA synthetase gene causes progressive spastic paresis instead of HUPRA syndrome: Hum Mutat. 2016;37(9):884888.

11. Riley LG, Cooper S, Hickey P, et al. Mutation of the mitochondrial tyrosyl-tRNA synthetase gene, YARS2, causes myopathy, lactic acidosis, and sideroblastic anemia - MLASA syndrome. Am J Hum Genet. 2010; 87(1):52-59

12. Riley LG, Rudinger-Thirion J, Schmitz-Abe K, et al. LARS2 variants associated with hydrops, lactic acidosis, sideroblastic anemia, and multisystem failure. JIMD. 2015;28:49-57.

13. Fontenay M, Cathelin S, Amiot M, Gyan E, Solary E. Mitochondria in hematopoiesis and hematological diseases. Oncogene. 2006;25(34):47574767

14. Testa U. Apoptotic mechanisms in the control of erythropoiesis. Leukemia. 2004;18(7):1176-1199. 